\title{
Transmission blocking activity of Azadirachta indica and Guiera senegalensis extracts on the sporogonic development of Plasmodium falciparum field isolates in Anopheles coluzzii mosquitoes
}

Rakiswendé S Yerbanga ${ }^{1 *}$, Leonardo Lucantoni ${ }^{2}$, Robert K Ouédraogo ${ }^{1}$, Dari F Da ${ }^{1}$, Franck A Yao ${ }^{1}$, Koudraogo B Yaméogo ${ }^{1}$, Thomas S Churcher ${ }^{3}$, Giulio Lupidi², Orazio Taglialatela-Scafati ${ }^{4}$, Louis Clément Gouagna², Anna Cohuet ${ }^{5}$, George K Christophides ${ }^{6}$, Jean Bosco Ouédraogo ${ }^{1}$ and Annette Habluetzel ${ }^{2}$

\begin{abstract}
Background: Targeting the stages of the malaria parasites responsible for transmission from the human host to the mosquito vector is a key pharmacological strategy for malaria control. Research efforts to identify compounds that are active against these stages have significantly increased in recent years. However, at present, only two drugs are available, namely primaquine and artesunate, which reportedly act on late stage gametocytes.

Methods: In this study, we assessed the antiplasmodial effects of 5 extracts obtained from the neem tree Azadirachta indica and Guiera senegalensis against the early vector stages of Plasmodium falciparum, using field isolates. In an ex vivo assay gametocytaemic blood was supplemented with the plant extracts and offered to Anopheles coluzzii females by membrane feeding. Transmission blocking activity was evaluated by assessing oocyst prevalence and density on the mosquito midguts.

Results: Initial screening of the 5 plant extracts at $250 \mathrm{ppm}$ revealed transmission blocking activity in two neem preparations. Up to a concentration of $70 \mathrm{ppm}$ the commercial extract NeemAzal ${ }^{\circledR}$ completely blocked transmission and at $60 \mathrm{ppm}$ mosquitoes of 4 out of 5 replicate groups remained uninfected. Mosquitoes fed on the ethyl acetate phase of neem leaves at $250 \mathrm{ppm}$ showed a reduction in oocyst prevalence of 59.0\% $\left(\mathrm{Cl}_{95} 12.0\right.$ - 79.0; $\left.p<10^{-4}\right)$ and in oocyst density of $90.5 \%\left(\mathrm{Cl}_{95} 86.0-93.5 ; p<10^{-4}\right)$, while the ethanol extract from the same plant part did not exhibit any activity. No evidence of transmission blocking activity was found using G. senegalensis ethyl acetate extract from stem galls.
\end{abstract}

Conclusions: The results of this study highlight the potential of antimalarial plants for the discovery of novel transmission blocking molecules, and open up the potential of developing standardized transmission blocking herbal formulations as malaria control tools to complement currently used antimalarial drugs and combination treatments.

Keywords: Plasmodium falciparum, Gametocytes, Sporogonic stages, Plant extracts, Transmission-blocking drugs

\footnotetext{
* Correspondence: yrserge@yahoo.fr

${ }^{1}$ Institut de Recherche en Sciences de la Santé, 01 BP545 Bobo Dioulasso,

Burkina Faso

Full list of author information is available at the end of the article
} 


\section{Background}

Thanks to the advancements of knowledge and to a significant expansion of financial resources supporting malaria programmes, the burden of the disease has been significantly reduced over the last decade. An estimated 274 million cases and 1.1 million deaths have been averted and 50 countries with ongoing malaria transmission are on track to reduce the incidence of malaria cases by $75 \%$ by 2015 [1]. However, since current and prospective international disbursements for malaria control reach not even half of the estimated yearly US\$ 5.1 billion required to achieve universal coverage of malaria interventions [1], it is highly uncertain whether the international targets for reducing malaria cases and deaths can be attained in all stricken countries.

Moreover the progress achieved remains under threat from the development of artemisinin resistant parasites, which have already been detected in 4 countries of the South East Asia Region [1]. The likelihood of resistance emergence is increased with the spread of poor-quality drugs. A recent review of studies conducted in 7 Asian and 21 sub-Saharan African countries unveiled an alarming occurrence of falsified, substandard and degraded antimalarial drugs with about one third of the drug samples tested failing chemical analysis by pharmacopeia standards [2].

This scenario calls for the acceleration of the discovery of novel antiplasmodial molecules and the design and development of new combination drugs tailored to the pharmacological needs of malaria control (i.e. to cure and prevent the disease in individuals and control/eliminate transmission). Combination drugs including molecules effective against transmissible stages of the parasite have a key role to play both as a resistance containment strategy and to equip countries entering the malaria elimination phase with the required tools. Current ACTs based on artemisinin compounds which are active on asexual blood stages as well as gametocytes have been shown to have a beneficial impact on transmission [3-5] despite being only partially effective [6]. The parasite's success in escaping elimination by artemisinin based drugs is associated with the molecules limited efficacy against the mature sexual stages in the vertebrate host [7], the short half-life of artemisinins and their active metabolites [8] and the long persistence (2-3 weeks) of mature gametocytes. Indeed, mature gametocytes are difficult targets for drug attack, which makes it a challenge to devise drugs that interfere with its biochemical pathways. As illustrated by early studies of RE Sinden [9] immature gametocytes are sensitive to inhibitors of DNA, RNA and protein synthesis, including antimalarials, but later stages to a minor extent. A treatment scheme with two different gametocytocidal molecules can be a practical approach, as shown by the study of Shekalaghe and colleagues [6] in which they showed that the administration of primaquine after a treatment course of sulphadoxine/pyrimethamine and artesunate caused an additional decrease in gametocyte densities to less than 0.1 per microliter of blood, a density at which the probability of mosquito infection is drastically reduced.

From a parasitologist's perspective, there are several arguments to support focusing on the early sporogonic stages developing in the midgut lumen of the mosquito host. First, transition from the vertebrate to the phylogenetically completely different mosquito host is an extremely critical phase in the parasite's life cycle. This may well explain why even without the introduction of an intervention the yield of the sexual process is so low. In rodent plasmodia it has been estimated that, from 500 macrogametocytes counted in the vertebrate host, only one goes through the whole process and develops into an oocyst on the midgut wall [10]. After blood ingestion by the mosquito, the sexual parasite stages undergo profound biological transformation and maturation within a few hours, involving numerous cellular and biochemical processes that offer plentiful potential drug targets [11]. Also, during this phase the parasites are extracellular, i.e. they lack protection by a host cell membrane, which facilitates drug interference with the parasite.

On this background it is not surprising that a large number of schizonticidal drugs designed to target asexual erythrocytic forms were found to display activity, and in some cases multi-stage effects, against the various transmissible Plasmodium stages [12]. For example, endoperoxides, such as OZ439, a synthetic molecule currently under clinical phase IIa trials, is a strong inhibitor of gametocyte maturation, gamete formation and has an impact on sporogony; lumefantrine and NPC-1161B, a new 8-aminoquinoline, also inhibits sporogony [12].

Learning the lesson from ACTs, i.e. the successful development of highly effective artemisinin based combination drugs starting from the medicinal plant Artemisia annua, prompted us to explore plants frequently used as anti-malarial remedies for possible transmission blocking effects. Among the plant species investigated, extracts from Azadirachta indica and Guiera senegalensis revealed in vivo transmission blocking activity (Plasmodium berghei, Anopheles stephensi, BALB/c mice) and/or in vitro inhibitory effects on the early sporogonic development of $P$. berghei. The Azadirachta indica extract, NeemAzal ${ }^{\oplus}$, tested in vitro at $6.5 \mu \mathrm{g} / \mathrm{ml}$ resulted in a $65.8 \%$ inhibition of early sporogonic stage development and a Guiera senegalensis ethylacetate fraction of galls tested at $50 \mathrm{ug} / \mathrm{ml}$ showed a $66 \%$ to $88 \%$ inhibitory activity (unpublished results). In particular, NeemAzal ${ }^{\oplus}$ a commercial (Trifolio-M $\mathrm{GmbH}$, Lahnau, Germany) methanol extract from seed kernels of $A$. indica rich in Azadirachtin A, showed prominent effects, completely inhibiting mosquito infection when 
administered intraperitoneally at a dosage of $50 \mathrm{mg} / \mathrm{kg}$ to gametocytaemic mice $[13,14]$.

The present study was undertaken to assess the activity of these plants on the transmissible stages of the human malaria parasite under field-like conditions. P. falciparum gametocytes from naturally infected humans and Anopheles coluzzii mosquitoes from colonies established in 2008 were used. Female mosquitoes were membrane fed with blood supplemented with plant extracts. In order to explore which compounds are likely to be responsible for transmission blocking activity, chemically characterized extracts prepared with various solvents $(\mathrm{MeOH}, \mathrm{EtOH}, \mathrm{EtOAc})$ from different plant parts were tested.

\section{Methods}

\section{Study area and recruitment of $P$. falciparum gametocyte carriers}

The study was conducted in the Bobo Dioulasso area (Burkina Faso) from May to October 2011. Malaria transmission is hyperendemic in the area, with an estimated P. falciparum entomological inoculation rate of 300-500 infective bites per person per year. Parasite prevalence and densities are strongly seasonal, with the transmission season peaking in September and lasting from approximately June to October. Gametocyte-positive blood samples for the direct membrane feeding assay (DMFA) were obtained from children, residents of the Dandé, Soumousso and Bama villages, situated at $40-55 \mathrm{~km}$ distance from Bobo-Dioulasso town.

A total of 2160 children aged $5-11$ years participated at 18 screening events organized from May to October 2011. For each event, groups of 120 children were invited to present at the village health center early in the morning. Every child was clinically examined for the presence of chronic diseases, acute infections other than malaria and signs of severe malaria. Finger-prick blood was collected and used for the preparation of thick smears. Information on anti-malarial drugs taken during the preceding 2 weeks as well as presence of hypersensitivity to anti-malarial drugs was recorded.

Thick smears were stained with Giemsa and examined in the laboratory on the same day. On each slide 100 fields were screened for the presence of Plasmodium parasites. Asexual parasite and gametocyte numbers were calculated per 200 and 1000 leukocytes respectively. Gametocytemia and parasitemia were then expressed as number of gametocytes or total number of parasites per microliter $(\mu \mathrm{L})$ of blood, assuming leukocyte counts of $8000 / \mu \mathrm{L}$ of blood.

Asymptomatic children with $P$. falciparum gametocytemia $\geq 56$ gametocytes $/ \mu \mathrm{L}$, parasitemia $\leq 1000$ parasites $/ \mu \mathrm{L}$ and negative for other Plasmodium species were selected as blood donors for the direct membrane feeding assay (DMFA) scheduled for the following day.
All children with confirmed malaria infection obtained treatment with the combination of artesunate $(4 \mathrm{mg} / \mathrm{kg}$ body weight)/amodiaquine (10 $\mathrm{mg} / \mathrm{kg}$ body weight), once daily for 3 days according to the guidelines of the National Malaria Control Programme. Children selected as gametocyte donors for the DMFA were treated the following morning after venous blood collection at the laboratory, those not recruited for the study were given the treatment the same evening by the village health workers.

\section{Anti-malarial plants and extracts}

Neem (A. indica A. Juss., Meliaceae) and G. senegalensis J. F. Gmel (Combretaceae), previously found to interfere with the sporogonic development of the rodent parasite P. berghei in Anopheles stephensi mosquitoes have been selected for this study $[13,14]$. The following plant parts and extracts were examined:

i) $\mathrm{NeemAzal}^{\circ}$ (NA), a commercial methanol extract from neem seed kernels containing azadirachtin A $34 \%$, other azadirachtins (azadirachtin B to K) $16 \%$, salannins 4\% and nimbins 2\% (Trifolio-M GmbH, Lahnau, Germany).

ii) two extracts of neem leaves collected in the Oubritenga Province, Burkina Faso, namely: a) total $\mathrm{EtOH}$ extract (NLE); b) EtOAc phase of the $\mathrm{EtOH}$ extract (NLA) obtained by partitioning NLE between water and EtOAc. The leaves contained limonoids, with gedunin as a major member of this class, but were devoid of azadirachtin. After partitioning, limonoids were concentrated in NLA.

iii) EtOAc phase of the total ethanol extract of neem fruits collected in the Oubritenga Province, Burkina Faso (NFA). This plant fraction has been subjected to detailed phytochemical analysis and 10 triterpenoid derivatives have been identified. The most abundant limonoids were azadirone and azadiradione accounting for $70 \%$ of the total components. The gedunin content was estimated at $3 \%$, azadirachtin was not present at detectable amounts [15].

iv) EtOAc extract of G. senegalensis stem galls, collected in the Bobo-Dioulasso area, Burkina Faso (GS).

Leaves and fruits of $A$. indica and G. senegalensis had been collected in the central region of Burkina Faso in June 2008. Plants were identified by Prof. Jeanne Millogo, professor of botanics at the Life Science Unit (University of Ouagadougou) and voucher specimen $\mathrm{N}^{\circ} 2 \mathrm{NFE}$ (A. indica), $\mathrm{N}^{\circ} 1$ GSE (G. Senegalensis) have been deposited in the Laboratory of Ecology at the University of Ouagadougou. Extracts were prepared and chemically characterized [13-15] at the Department of Pharmacy of the University of Naples "Federico II". 
All extracts were initially screened at the high dosage of $250 \mathrm{ppm}$ to allow any possible transmission blocking effects to be detected even by compounds present at low concentrations. Extracts found active were then tested at decreasing doses.

\section{Transmission blocking experiments}

The transmission blocking efficacy of the plant extracts on $P$. falciparum gametocyte field isolates was assessed on $A n$. coluzzii mosquitoes, using a colony established in 2008 from field collected mosquitoes. For the membrane feeding assays, 4-5 day old females, kept without sucrose solution for 24 hours before the experimental infection, were used.

Approximately $8 \mathrm{~mL}$ of gametocytaemic blood was collected from each selected child by venous puncture using heparinized tubes. Care was taken to keep the blood tubes constantly at $37^{\circ} \mathrm{C}$ to avoid gametocyte activation leading to precocious gamete formation. Volumes of $10-35 \mu \mathrm{L}$ of experimental extracts, dissolved in sterile distilled water for injection were added to $1 \mathrm{~mL}$ blood aliquots to obtain the desired final extract concentrations. Equal amounts of sterilized distilled water for injection were added to the control blood samples. Extract supplements and control blood mixtures were transferred to membrane feeders and 50 mosquito females per feeder were allowed to take blood for 30 to 60 minutes. Fed mosquitoes were separated from the unfed specimens and kept on a $10 \%$ sucrose diet. On day 7 after membrane feeding, midguts were dissected from all surviving females, stained with 1\% mercurochrome in PBS (phosphate buffered saline, $\mathrm{pH}$ 7.2) and the presence and number of oocysts recorded for each mosquito. The transmission blocking activity of the plant extracts was estimated by determining both oocyst prevalence and oocyst density. For each test extract and concentration, two to five independent replicates were performed and prevalence and density values calculated for the treatment and control groups.

\section{Statistical analysis}

Generalized linear mixed models were used to compare mosquito infection in control and treatment groups and estimate intervention efficacy [16]. Differences in oocyst prevalence (number of oocyst positive mosquitoes per group) were tested assuming the number of positive mosquitoes followed a binomial distribution. The number of oocysts per mosquito (including negatives mosquitoes) was best described using a zero-inflated negative binomial distribution. Ninety-five percent confidence interval estimates were generated using bootsrapping methodology.

\section{Ethical approval}

The study was approved by the ethical committee of the Centre Muraz and filed under the registration number
N/Ref. 003-2009/CE-CM. Parents or guardians provided written informed consent before children were enrolled into the blood collection protocol.

\section{Results}

The transmission blocking activity of the four $A$. indica extracts and the $G$. senegalensis extract was investigated in a series of 31 membrane feeding experiments using P. falciparum gametocyte-positive blood from 18 different donors. Gametocyte densities varied among the experimental blood samples from 56 (threshold for inclusion) to 1760 sexual forms per microliter of blood; two thirds of the samples (12/18) displayed gametocyte densities between 96 and 416 (Table 1). Infection of control mosquitoes varied considerably between the experiments: oocyst prevalence ranged between $45 \%$ and $92 \%$ (mean $70 \%, \mathrm{CI}_{95} 65.7-74.7$ ) and oocyst densities between 1.4 and 151.6 (mean 31.5, $\mathrm{CI}_{95}$ 17.1-45.8; Tables 2 and 3).

Initial screening of the plant extracts at $250 \mathrm{ppm}$ revealed transmission blocking activity in two out of the four A. indica preparations (Table 2). At this high dose, the

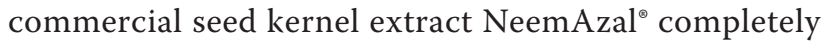
blocked transmission. The total ethanol extract from the neem leaves did not exhibit any activity (Table 2, Figure 1), while the EtOAc phase of this extract reduced oocyst prevalence by $59.0 \%\left(\mathrm{CI}_{95} 12.0-79.0 ; p<10^{-4}\right)$ and oocyst density by $90.5 \%\left(\mathrm{CI}_{95} 86.0-93.5 ; p<10^{-4}\right)$. The mean

Table 1 Blood samples used for membrane feeding assays

\begin{tabular}{|c|c|c|c|}
\hline $\begin{array}{l}\text { Blood } \\
\text { sample } \\
\text { number }\end{array}$ & $\begin{array}{l}\text { Hemoglobin } \\
(\mathbf{g} / \mathrm{dL})\end{array}$ & $\begin{array}{l}\text { Plasmodium } \\
\text { falciparum } \\
\text { (asexual forms) } \\
\text { per microliter }\end{array}$ & $\begin{array}{c}\text { Plasmodium falciparum } \\
\text { gametocytes } \\
\text { (sexual forms) } \\
\text { per microliter }\end{array}$ \\
\hline 1 & 15.0 & 0 & 56 \\
\hline 2 & 15.0 & 0 & 104 \\
\hline 3 & 15.4 & 1040 & 120 \\
\hline 4 & 15.2 & 0 & 128 \\
\hline 5 & 13.4 & 400 & 216 \\
\hline 6 & 15.1 & 0 & 528 \\
\hline 7 & 14.8 & 0 & 112 \\
\hline 8 & 15.2 & 560 & 96 \\
\hline 9 & 12.2 & 800 & 64 \\
\hline 10 & 15.6 & 400 & 232 \\
\hline 11 & 14.7 & 0 & 400 \\
\hline 12 & 14.0 & 0 & 1760 \\
\hline 13 & 14 & 1440 & 64 \\
\hline 14 & 12.1 & 80 & 416 \\
\hline 15 & 12.9 & 880 & 344 \\
\hline 16 & 13.5 & 480 & 184 \\
\hline 17 & 14.1 & 2000 & 112 \\
\hline 18 & 9.3 & 0 & 88 \\
\hline
\end{tabular}


Table 2 Transmission blocking activity of Azadirachta indica and Guiera senegalensis extracts at 250 ppm (initial screening dosage) on Plasmodium falciparum oocyst

\begin{tabular}{|c|c|c|c|c|}
\hline $\begin{array}{l}\text { Blood sample } \\
\text { number }\end{array}$ & $\begin{array}{l}\text { Gametocytes } \\
\text { per } \mu \mathrm{L} \text { blood }\end{array}$ & $\begin{array}{l}\text { Treatment }{ }^{*} \text { and } \\
\text { concentration }\end{array}$ & $\begin{array}{c}\text { Prevalence of infected mosquitoes\% } \\
\text { (infected/total examined) }\end{array}$ & $\begin{array}{c}\text { Number of } \\
\text { oocysts/mosquito }\left(\mathrm{Cl}_{95}\right) \\
\end{array}$ \\
\hline \multirow[t]{4}{*}{1} & \multirow[t]{4}{*}{56} & control & $53(10 / 19)$ & $1.79(0.30-3.28)$ \\
\hline & & NA 250 ppm & $0(0 / 22)$ & 0.00 \\
\hline & & control & $58(15 / 26)$ & $3.23(1.34-5.12)$ \\
\hline & & NA 250 ppm & $0(0 / 15)$ & 0.00 \\
\hline \multirow{2}{*}{9} & \multirow{2}{*}{64} & control & $71(39 / 55)$ & $7.15(5.10-9.19)$ \\
\hline & & NLA 250 ppm & $20(09 / 44)$ & $0.32(0.09-0.54)$ \\
\hline \multirow{2}{*}{13} & \multirow{2}{*}{64} & control & $54(21 / 39)$ & $1.95(0.87-3.02)$ \\
\hline & & NLA 250 ppm & $12(05 / 42)$ & $0.26(0.00-0.52)$ \\
\hline \multirow{2}{*}{4} & \multirow{2}{*}{128} & control & $55(18 / 33)$ & $1.91(0.91-2.91)$ \\
\hline & & NLA 250 ppm & $35(08 / 23)$ & $0.57(0.14-0.99)$ \\
\hline \multirow{2}{*}{10} & \multirow{2}{*}{232} & control & $76(32 / 42)$ & $15.69(10.82-20.56)$ \\
\hline & & NLA 250 ppm & $76(13 / 22)$ & $1.36(0.54-2.19)$ \\
\hline \multirow{2}{*}{13} & \multirow{2}{*}{64} & control & $45(15 / 33)$ & $1.39(0.59-2.20)$ \\
\hline & & NLE 250 ppm & $42(13 / 43)$ & $2.67(1.35-4.00)$ \\
\hline \multirow{2}{*}{5} & \multirow{2}{*}{216} & control & $53(09 / 17)$ & $18.88(4.26-33.50)$ \\
\hline & & NLE 250 ppm & $65(15 / 23)$ & $27.83(10.20-39.45)$ \\
\hline \multirow{2}{*}{10} & \multirow{2}{*}{232} & control & $71(35 / 49)$ & $10.61(7.16-14.06)$ \\
\hline & & NLE 250 ppm & $84(31 / 37)$ & $20.92(14.50-27.34)$ \\
\hline \multirow{2}{*}{18} & \multirow{2}{*}{88} & control & $56(14 / 25)$ & $2.28(0.58-3.98)$ \\
\hline & & NFA 250 ppm & $10(03 / 30)$ & $0.20(0.00-0.45)$ \\
\hline \multirow{2}{*}{3} & \multirow{2}{*}{120} & control & $67(32 / 48)$ & $3.69(2.28-5.09)$ \\
\hline & & NFA 250 ppm & $41(29 / 70)$ & $1.84(1.03-2.65)$ \\
\hline \multirow{2}{*}{14} & \multirow{2}{*}{416} & control & $66(31 / 32)$ & 63.59 (37.99-89.19) \\
\hline & & NFA 250 ppm & $88(30 / 34)$ & $88.15(60.08-116.21)$ \\
\hline \multirow{2}{*}{8} & \multirow{2}{*}{96} & control & $61(25 / 41)$ & $1.90(1.11-2.70)$ \\
\hline & & GS 250 ppm & $58(21 / 36)$ & $2.14(1.05-3.23)$ \\
\hline \multirow{2}{*}{2} & \multirow{2}{*}{104} & control & $58(21 / 36)$ & $6.67(3.74-9.59)$ \\
\hline & & GS 250 ppm & $41(09 / 22)$ & $2.05(0.63-3.46)$ \\
\hline \multirow{2}{*}{7} & \multirow{2}{*}{112} & control & $71(27 / 38)$ & $2.71(1.55-3.87)$ \\
\hline & & GS 250 ppm & $71(36 / 51)$ & $4.61(3.14-6.07)$ \\
\hline
\end{tabular}

${ }^{\circ} \mathrm{NA}:$ NeemAzal ${ }^{\circ}$; NLA: neem leaves EtOAc extract; NLE: neem leaves EtOH extract; NFA: neem fruits EtOAc extract; GS: Guiera senegalensis stem gall EtOAc extract.

percentage inhibition for each experiment compared to its control from the four independent replicates was respectively $96.0 \%\left(\mathrm{CI}_{95} 94.0\right.$ - 98.0), 87.0\% $\left(\mathrm{CI}_{95} 83.0\right.$ - 99.6), $70.0 \%\left(\mathrm{CI}_{95} 66.0-85.0\right)$ and $91.0 \%\left(\mathrm{CI}_{95} 89.0\right.$ - 95.0). The neem fruit EtOAc extract showed inconsistent results: in two experiments using blood with relatively low gametocytemia (120 and 88 gametocytes $/ \mu \mathrm{L}$ ) oocyst prevalence and density were reduced by $58.54 \%\left(\mathrm{CI}_{95} 22.69-90.38\right.$, $p=0.007)$ and $65.83 \%\left(\mathrm{CI}_{95} 63.98-65.82, p=0.008\right)$ in treated mosquitoes compared to controls. The third replicate experiment however, performed with a blood sample rich in gametocytes (416 gametocytes/ $\mu \mathrm{L}$ ) yielded numerous oocysts in both, treated and control mosquitoes
(Table 2). No evidence of transmission blocking activity was found with the G. senegalensis EtOAc extract from stem galls.

When $\mathrm{NeemAzal}^{\oplus}$ was tested at decreasing dosages it completely blocked mosquito infection at $70 \mathrm{ppm}$ in two independent replicates, at $60 \mathrm{ppm}$ in four out of five, and at $50 \mathrm{ppm}$ in two out of five replicate experiments (Table 3). At 50 ppm NeemAzal ${ }^{\bullet}$ treatment (5 replicates), a strong impact on both oocyst prevalence and density was observed: $15.4 \%\left(\mathrm{CI}_{95} 0.00-34.67\right)$ of the treatment group were found positive for oocyst as compared to $81.6 \%$ $\left(\mathrm{CI}_{95}\right.$ 65.16-98.04) of control mosquitoes, corresponding to a reduction of $79.9 \%\left(\mathrm{CI}_{95} 58.72-81.29 ; p<10^{-6}\right)$ 
Table 3 Transmission blocking activity: dose dependent efficacy of NeemAzal ${ }^{\circledR}$ (NA) on Plasmodium falciparum oocyst prevalence and density

\begin{tabular}{|c|c|c|c|c|}
\hline $\begin{array}{l}\text { Blood sample } \\
\text { number }\end{array}$ & $\begin{array}{l}\text { Gametocytes } \\
\text { per } \mu \mathrm{L} \text { blood }\end{array}$ & $\begin{array}{l}\text { Treatment and } \\
\text { concentration }\end{array}$ & $\begin{array}{l}\text { Prevalence of infected mosquitoes\% } \\
\text { (infected/total examined) }\end{array}$ & $\begin{array}{c}\text { Mean number of } \\
\text { oocysts/mosquito }\left(\mathrm{Cl}_{95}\right)\end{array}$ \\
\hline \multirow{2}{*}{11} & \multirow{2}{*}{400} & control & $64(09 / 14)$ & 51.36 (12.83-89.88) \\
\hline & & NA 70 ppm & $0(0 / 25)$ & 0.00 \\
\hline \multirow{2}{*}{12} & \multirow{2}{*}{1760} & control & $75(12 / 16)$ & 94.38 (38.29-150.46) \\
\hline & & NA 70 ppm & $0(00 / 25)$ & 0.00 \\
\hline \multirow{2}{*}{17} & \multirow{2}{*}{112} & control & $80(12 / 15)$ & $4.53(2.69-6.38)$ \\
\hline & & NA 60 ppm & $0(0 / 31)$ & 0.00 \\
\hline \multirow{2}{*}{16} & \multirow{2}{*}{184} & control & $64(29 / 45)$ & $4.58(2.96-6.19)$ \\
\hline & & NA 60 ppm & $0(0 / 56)$ & 0.00 \\
\hline \multirow{2}{*}{15} & \multirow{2}{*}{344} & control & $90(09 / 10)$ & $131.00(49.69-212.31)$ \\
\hline & & NA 60 ppm & $12(03 / 25)$ & $0.36(0.00-0.83)$ \\
\hline \multirow{2}{*}{11} & \multirow{2}{*}{400} & control & $68(17 / 25)$ & $49.36(22.92-75.80)$ \\
\hline & & NA 60 ppm & $0(0 / 25)$ & 0.00 \\
\hline \multirow{2}{*}{12} & \multirow{2}{*}{1760} & control & $75(12 / 16)$ & 94.38 (38.29-150.46) \\
\hline & & NA 60 ppm & $0(0 / 25)$ & 0.00 \\
\hline \multirow{2}{*}{17} & \multirow{2}{*}{112} & control & $85(11 / 13)$ & $3.92(1.91-5.93)$ \\
\hline & & NA 50 ppm & $23(07 / 31)$ & $0.35(0.05-0.66)$ \\
\hline \multirow{2}{*}{16} & \multirow{2}{*}{184} & control & $59(24 / 41)$ & $2.68(1.58-3.79)$ \\
\hline & & NA 50 ppm & $0(0 / 46)$ & 0.00 \\
\hline \multirow{2}{*}{15} & \multirow{2}{*}{344} & control & $92(24 / 26)$ & 151.58 (97.31-205.84) \\
\hline & & NA 50 ppm & $36(15 / 42)$ & $2.29(0.60-3.97)$ \\
\hline \multirow{2}{*}{11} & \multirow{2}{*}{400} & control & $82(28 / 34)$ & $66.50(44.04-88.96)$ \\
\hline & & NA 50 ppm & $0(0 / 23)$ & 0.00 \\
\hline \multirow{2}{*}{6} & \multirow{2}{*}{528} & control & $90(54 / 60)$ & 42.85 (31.36-54.34) \\
\hline & & NA 50 ppm & $18(11 / 61)$ & $0.30(0.10-0.49)$ \\
\hline \multirow{2}{*}{17} & \multirow{2}{*}{112} & control & $67(18 / 27)$ & $6.56(3.60-9.51)$ \\
\hline & & NA 20 ppm & $52(12 / 23)$ & $2.57(0.86-4.27)$ \\
\hline \multirow{2}{*}{16} & \multirow{2}{*}{184} & control & $84(38 / 45)$ & $4.42(2.92-5.93)$ \\
\hline & & NA 20 ppm & $41(26 / 63)$ & $0.95(0.56-1.34)$ \\
\hline \multirow{2}{*}{15} & \multirow{2}{*}{344} & control & $81(29 / 36)$ & $92.69(47.92-137.46)$ \\
\hline & & NA 20 ppm & $60(12 / 20)$ & $44.85(7.90-81.80)$ \\
\hline 6 & 520 & control & $85(44 / 52)$ & $32.90(22.36-43.45)$ \\
\hline 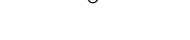 & 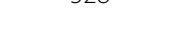 & NA 20 ppm & $88(44 / 50)$ & $27.24(19.12-35.36)$ \\
\hline
\end{tabular}

(Figure 2). On NeemAzal ${ }^{\oplus}$-treated mosquito midguts a mean of $0.62\left(\mathrm{CI}_{95} 0.25-0.98\right)$ oocysts was recorded, 99.06\% $\left(\mathrm{CI}_{95} 98.52-99.40 ; p<10^{-6}\right)$ less than on control midguts (51.34, $\mathrm{CI}_{95} 39.39$ - 63.30; Table 3, Figure 2). At 20 ppm, oocyst-positive mosquitoes were observed in each of the 4 treatment group replicates and the mean oocyst prevalence was not different in the $\mathrm{NeemAzal}^{\oplus}$ $\left(60.25 \%, \mathrm{CI}_{95} 28.31\right.$ - 92.19) group with respect to the control groups $\left(79.25 \%, \mathrm{CI}_{95} 65.98\right.$ - 92.52; $\left.\mathrm{p}=0.17\right)$. However, analysis of oocyst densities still evidenced a reduction of $52.69 \%\left(\mathrm{CI}_{95} 34.81-65.67 ; p<10^{-6}\right.$; Table 3 and Figure 3).

\section{Discussion}

Extracts from $A$. indica (neem) were found to contain components with transmission blocking activity against $P$. falciparum field isolates in our vector infection experiments conducted in Burkina Faso. Of the different $A$. indica extracts examined, NeemAzal ${ }^{\circ}$, an azadirachtin-enriched preparation of neem seeds showed prominent inhibitory activity on parasite development in the vector. When added at a concentration of $60 \mathrm{ppm}$ to gametocytaemic blood from $P$. falciparum infected donors and membrane fed to An. coluzzii females, oocyst development was completely suppressed in four out of five independent replicates. At 


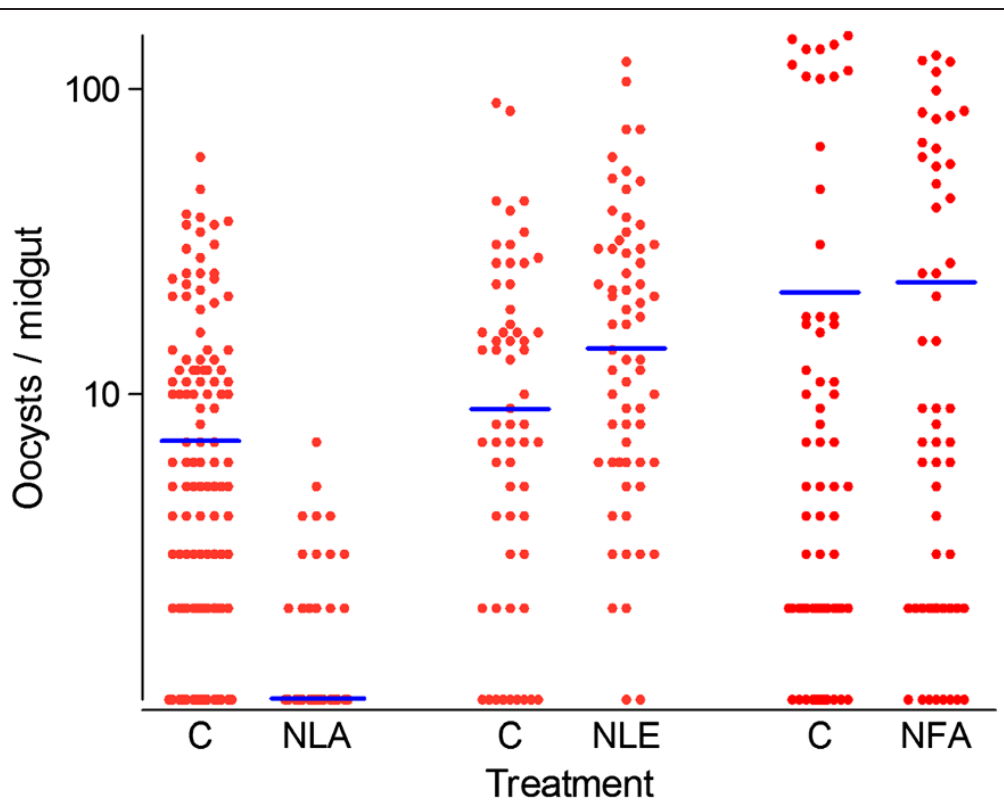

Figure 1 Effect of $250 \mathrm{ppm}$ Neem extracts on oocyst formation of $P$. falciparum field isolates in An. coluzzi mosquitoes. NLA (leaves EtOAC extract, 4 independent replicates), NLE (leaves EtOH extract, 3 independent replicates) and NFA (fruits EtOAc extract, 3 independent replicates), C (controls). Each point represents the number of oocysts per mosquito (including mosquitoes with zero oocysts); horizontal bars (blue lines) indicate geometric means calculated on all mosquitoes (negatives included).

$50 \mathrm{ppm}$, oocyst prevalence was reduced by $80 \%$ and density by $99 \%$ and at $20 \mathrm{ppm}$, a $53 \%$ decrease in oocyst density was still observed.

Despite considerable variation of gametocyte density between replicates, consistent dose dependent results were obtained, substantiating the specificity of the NeemAzal ${ }^{\circ}$ action and providing support for the robustness of the
P. falciparum field isolate membrane feeding assay developed at IRSS in Bobo-Dioulasso. These results establish the transmission blocking activity of NeemAzal ${ }^{\circ}$ against the human parasite $P$. falciparum, and are in excellent agreement with the previously reported activity in the murine parasite model P. berghei and An. stephensi mosquitoes [14].

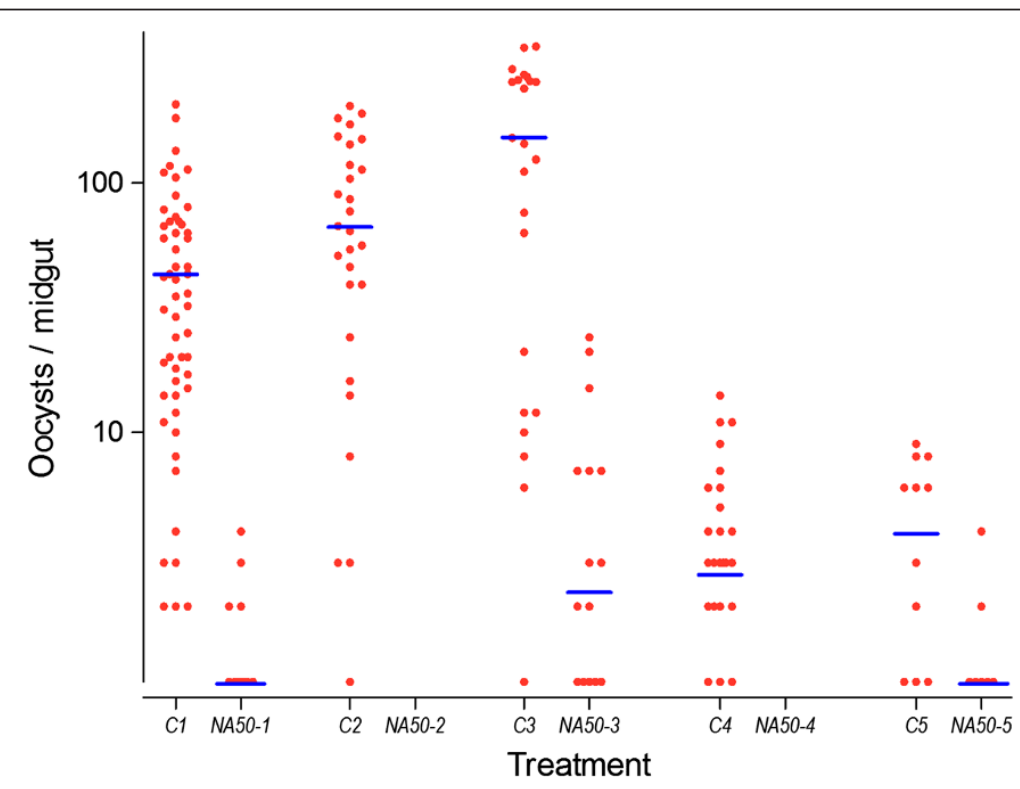

Figure 2 Effect of $50 \mathrm{ppm}$ NeemAzal ${ }^{\circledR}$ on sporogonic development to oocyst formation (5 independent replicates). Control groups: C1- C5, treatment groups: NA50-1 to NA50-5. Each point represents the number of oocysts per mosquito (including mosquitoes with zero oocysts); horizontal bars (blue lines) indicate geometric means calculated from all mosquitoes (negatives included). 


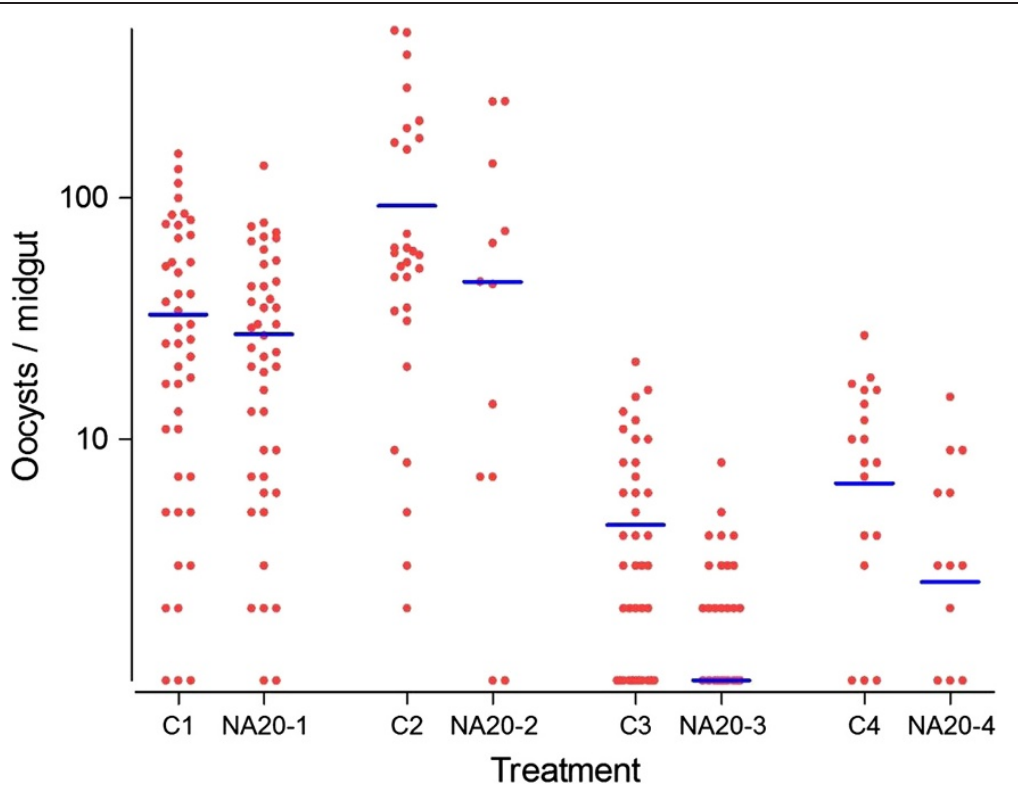

Figure 3 Effect of 20 ppm NeemAzal ${ }^{\circledR}$ on sporogonic development of oocyst formation (4 independent replicates). Control groups: C1C4, treatment groups: NA20-1 - NA20-4. Each point represents the number of oocysts per mosquito (including mosquitoes with zero oocysts); horizontal bars (blue lines) indicate geometric means calculated from all mosquitoes (negatives included).

The neem tree $($ A. indica) is a popular medicinal plant used in various Asian and African countries for the cure of various ailments and illnesses caused by infectious agents, including malaria. More frequently leaves, but also fruits, seeds and the bark are employed for the preparation of traditional remedies [13-17]. The efficacy of these preparations has been associated with the large number of secondary metabolites present in the plant, including at least 50 bioactive limonoids [18]. Studies aimed at detecting the molecules responsible for the antimalarial activity of neem extracts found that the limonoids nimbolide and gedunin displayed major inhibitory effects on in vitro growth of $P$. falciparum asexual blood stages [19-22]. Azadirachtin, moderately active on asexual blood stages [23], has been demonstrated to inhibit the exflagellation process of $P$. falciparum and $P$. berghei microgametocytes in vitro, the initial phase of sporogonic development in the vector $[24,25]$. In studies conducted with the azadirachtin-enriched standardized extract $\mathrm{NeemAzal}^{\circ}$, the product was found to completely block the development of P. berghei in An. stephensi mosquitoes when fed on gametocytemic mice previously treated with $\mathrm{NeemAzal}^{\circ}$ at an azadirachtin concentration of $50 \mathrm{mg} / \mathrm{kg}$ body weight [14]. The reduced number of ookinetes recorded in the NeemAzal ${ }^{\circ}$ treated group suggested an interference with the parasite development already before zygote formation and provides indirect evidence of azadirachtin interference with microgametogenesis in vivo. In addition, post-zygotic forms from $\mathrm{NeemAzal}^{\circledR}$ treated mosquitoes displayed evident morphological alterations and no mature ookinetes could be detected at the $50 \mathrm{mg} / \mathrm{kg}$ dosage, indicating that NeemA$\mathrm{zal}^{\circ}$ action is targeted to the early sporogonic stages developing in the midgut lumen. This assumption was further supported by the observation that NeemAzal ${ }^{\circ}$, when blood fed to already infected mosquitoes did not interfere with oocyst development [14].

Several studies report that azadirachtin and other limonoids present in neem extracts are active on mosquitoes $[26,27]$. Exploring the insecticidal activity of $\mathrm{NeemAzal}^{\circ}$ in An. stephensi, it emerged that females membrane fed with NeemAzal ${ }^{\circ}$ containing bloodmeals at an azadirachtin concentration of $100 \mu \mathrm{g} / \mathrm{mL}$ displayed reduced blood feeding capability and produced fewer eggs [28]. This reduction in oviposition was mirrored by degenerative tissue damage in ovarian follicles [28]. In addition, studies in laboratory animals indicated a very low acute toxicity of NeemAzal ${ }^{\circ}$ by all routes tested; lethal dose for $50 \%$ of the animal population $\left(\mathrm{LD}_{50}\right)$ was higher to $5000 \mathrm{mg} / \mathrm{kg}$ body weight in rat [29].

In this study, a transmission blocking activity on $P$. falciparum field isolates was also displayed by the EtOAc fraction from neem leaves (NLA). Added at $250 \mathrm{ppm}$ to blood from gametocytaemic donors and membrane fed to An. coluzzii mosquitoes, NLA reduced oocyst prevalence by $59 \%$ and oocyst intensity by $90 \%$. Since this extract does not contain detectable levels of azadirachtin, this activity must be attributed to the presence of other neem components. Gedunin, which is abundant in NLA might be involved. This molecule, previously reported to possess gametocytocidal activity in vitro [30], certainly deserves attention for further studies. Comparison of the 
results obtained with the total neem leaf ethanol extract and its EtOAc fraction (NLA, no evidence of transmission blocking activity in the first and in the second replicate), allows us to assume that the unidentified active molecules have apolar characteristics. This feature, together with the abundance of limonoids in NLA suggests their belonging to the limonoid class.

The examined EtOAc extract from neem fruits did not show consistent transmission blocking inhibitory effects at $250 \mathrm{ppm}$ initial screening dose. Detailed phytochemical analysis of this plant fraction allowed the isolation of 10 triterpenoid derivatives including the identification of two new limonoid molecules named neemfruitins A and B [15]. Since the major components of this the extract were identified as azadirone and azadiradione (70\% of the total components), a transmission blocking activity can be, most likely, excluded for these two molecules. Azadirachtin was not present in detectable amounts and gedunin accounted for only $3 \%$ of the total neem fruit limonoids [15] and could potentially be responsible for the partial activity displayed. Interestingly, in vitro screening for anti-blood stage activity using a $P$. falciparum chloroquine sensitive (D10) and resistant (W2) strain revealed prominent in vitro schizontocidal activity of the EtOAc neem fruit extract. $\mathrm{IC}_{50}$ values of $1.31-3.35 \mu \mathrm{g} / \mathrm{mL}$ were found. Similar values were observed for the isolated molecules azadirone, gedunin and neemfruitin A [15]. Given the relatively low abundance of highly active limonoids in these extracts, their prominent activity cannot be explained only on the basis of additive effects, but a synergistic action between the constituent molecules should be taken into account [15]. In addition, the in vivo activity of neem fruits has been demonstrated: mice treated at a daily oral dosage of $200 \mathrm{mg} / \mathrm{kg}$ crude extract over 9 days and exposed to infectious mosquito bites on day 3 of treatment displayed parasitaemia levels reduced by $45 \%$ [17].

Stem gall ethyl acetate extract from G. senegalensis, the second anti-malarial plant selected for this study, did not display transmission blocking activity. It has been included in this study since remedies based on the galls of this plant are frequently used in the Bobo-Dioulasso area for the treatment of fevers and similarly to neem, G. senegalensis contains abundant terpenoids and phenolic compounds (tannins, flavonoids) [31].

\section{Conclusion}

In conclusion, this study confirmed activity of limonoidrich medicinal plant extracts on the sporogonic development of P. falciparum isolates in An. coluzzii mosquitoes. The results strongly suggest azadirachtin to be an important compound, but the results also provide evidence for the presence of at least one other molecule with transmission blocking activity. Screening many purified molecules would have not been practical in this study because of logistical and ethical limitations (e.g. distance from the field to the lab; amount of blood that can be collected from individual donors, especially children). However, by focusing on a small panel of extracts with different limonoid content profiles it was possible to deduce useful information on the transmission blocking activity of single constituent molecules.

Considering the anti-plasmodial activity of neem limonoids on different life cycle stages of the parasite these compounds hold promise for the design of new, effective, multi-stage combination medicines. As an example, herbal medicines designed as preventivetransmission blocking formulations, if used by entire communities, may reduce incidence of malaria cases and decrease the intensity of transmission. Studies aimed at assessing bioavailability of pure azadirachtin and azadirachtin rich preparations have been initiated in order to validate the feasibility of the approach.

\section{Competing interests}

I declare that no competing interests existed for the authors or the institute before, during and after preparing and submitting this paper for review.

\section{Authors' contributions}

RSY participated in study design, carried out the experiments, performed the statistical analysis, and drafted the manuscript; LL participated in study design; RKO, DFA, FAY and KBY participated in the execution of the experiments; TSC: conducted the statistical analysis; RSY, GL and OTS participated and helped with plant extraction and partitioning; LCG, AC and GKC participated in study design and helped with manuscript revision; JBO and $\mathrm{AH}$ coordinated the work, participated in study design and critically revised the manuscript. All the authors read and approved the final manuscript.

\section{Acknowledgments}

We acknowledge the following colleagues for critically reviewing the manuscript: Robert Sinden, Imperial College of London (UK); Fulvio Esposito, University of Camerino, Camerino(IT). Trifolio-M GmbH, Lahnau, Germany for providing us with NeemAzal ${ }^{\oplus}$. The work was financially supported by the Seventh European Framework Programme project TransMalariaBloc' n. 223736.

\section{Author details}

${ }^{1}$ Institut de Recherche en Sciences de la Santé, 01 BP545 Bobo Dioulasso, Burkina Faso. ${ }^{2}$ Scuola di Scienze del Farmaco e dei Prodotti della Salute, Università di Camerino, 62032 Camerino, MC, Italy. ${ }^{3}$ Department of Infectious Disease Epidemiology, Imperial College London, London W2 1PG, United Kingdom. "Dipartimento di Farmacia, Università di Napoli "Federico II", via D. Montesano, 49, I-80131 Napoli, Italy. Institut de Recherche pour le Développement, Unité MIVEGEC (IRD 224- CNRS 5290-UM1-UM2), BP 64501 Montpellier Cedex 5 34394, France. ${ }^{6}$ Department of Life Sciences, Imperial College London, London, United Kingdom.

Received: 14 February 2014 Accepted: 10 April 2014 Published: 15 April 2014

\section{References}

1. World Health Organization: World malaria report 2012. Geneva: World Health Organization; 2012.

2. Nayyar GML, Breman JG, Newton PN, Herrington J: Poor-quality antimalarial drugs in southeast Asia and sub-Saharan Africa. Lancet Infect Dis 2012, 12:488-496.

3. Stepniewska K, Price RN, Sutherland CJ, Drakeley CJ, von Seidlein L, Nosten F, White NJ: Plasmodium falciparum gametocyte dynamics in areas of different malaria endemicity. Malar J 2008, 7:249.

4. Bousema T, Okell L, Shekalaghe S, Griffin JT, Omar S, Sawa P, Sutherland C, Sauerwein R, Ghani AC, Drakeley C: Revisiting the circulation time of 
Plasmodium falciparum gametocytes: molecular detection methods to estimate the duration of gametocyte carriage and the effect of gametocytocidal drugs. Malar J 2010, 9:136

5. Abay SM: Blocking malaria transmission to Anopheles mosquitoes using artemisinin derivatives and primaquine: a systematic review and meta-analysis. Parasite Vectors 2013, 6:278.

6. Shekalaghe S, Drakeley C, Gosling R, Ndaro A, van Meegeren M, Enevold A, Alifrangis M, Mosha F, Sauerwein R, Bousema T: Primaquine clears submicroscopic Plasmodium falciparum gametocytes that persist after treatment with sulphadoxinepyrimethamine and artesunate.

PLoS One 2007, 2:e1023.

7. Pukrittayakamee $\mathrm{S}$, Chotivanich $\mathrm{K}$, Chantra A, Clemens R, Looareesuwan S, White NJ: Activities of artesunate and primaquine against asexual and sexual stage parasites in falciparum malaria. Antimicrob Agents 2004, 48:1329-1334

8. Newton P, Suputtamongkol Y, Teja-Isavadharm P, Pukrittayakamee S, Navaratnam V, Bates I, White NJ: Antimalarial bioavailability and disposition of artesunate in acute falciparum malaria. Antimicrob Agents Chemother 2000, 44:972-977.

9. Sinden RE: Gametocytogenesis in Plasmodium spp., and observations on the meiotic division. Ann Soc Belg Med Trop 1985, 65(2):21-23.

10. Sinden RE, Dawes EJ, Alavi Y, Waldock J, Finney O, Mendoza J, Butcher GA, Andrews L, Hill AV, Gilbert SC, Basáñez MG: Progression of Plasmodium berghei through Anopheles stephensi is density-dependent. PLoS Pathog 2007, 3:e195.

11. Sinden RE, Carter R, Drakeley C, Leroy D: The biology of sexual development of Plasmodium: the design and implementation of transmission-blocking strategies. Malar J 2012, 11:70.

12. Delves M, Plouffe D, Scheurer C, Meister S, Wittlin S, Winzeler EA, Sinden RE, Leroy D: The activities of current antimalarial drugs on the life cycle stages of plasmodium: a comparative study with human and rodent parasites. PLoS Med 2012, 9:e1001169.

13. Yerbanga RS: Validation of traditional remedies employed to prevent malaria and identification of plants with potential to block the transmission. PhD thesis: School of advanced studies, Univervità di camerino; 2010.

14. Lucantoni L, Yerbanga RS, Lupidi G, Pasqualini L, Esposito F, Habluetzel A: Transmission blocking activity of a standardized neem (Azadirachta indica) seed extract on the rodent malaria parasite Plasmodium berghei in its vector Anopheles stephensi. Malar J 2010, 9:66.

15. Chianese G, Yerbanga SR, Lucantoni L, Habluetzel A, Basilico N, Taramelli D, Fattorusso E, Taglialatela-Scafati O: Antiplasmodial triterpenoids from the fruits of neem, Azadirachta indica. J Nat Prod 2010, 73:1448-1452.

16. Churcher TS, Blagborough AM, Delves M, Ramakrishnan C, Kapulu MC, Williams AR, Biswas S, Da DF, Cohuet A, Sinden RE: Measuring the blockade of malaria transmission - An analysis of the Standard Membrane Feeding Assay. Int J Parasitol 2012, 42:1037-1044.

17. Yerbanga RS, Lucantoni L, Lupidi G, Dori GU, Tepongning NR, Nikiéma JB, Esposito F, Habluetzel A: Antimalarial plant remedies from Burkina Faso: their potential for prophylactic use. J Ethnopharmacol 2012, 140:255-260

18. Roy A, Saraf S: Limonoids: overview of significant bioactive triterpenes distributed in plants kingdom. Biol Pharm Bull 2006, 29:191-201.

19. MacKinnon S, Durst T, Arnason JT, Angerhofer C, Pezzuto J, Sanchez-Vindas PE, Poveda LJ, Gbeassor M: Antimalarial activity of tropical Meliaceae extracts and gedunin derivatives. J Nat Prod 1997, 60:336-341.

20. Khalid SA, Farouk A, Geary TG, Jensen JB: Potential antimalarial candidates from African plants: and in vitro approach using Plasmodium falciparum. J Ethnopharmacol 1986, 15:201-209.

21. Rochanakij S, Thebtaranonth Y, Yenjai C, Yuthavong Y: Nimbolide, a constituent of Azadirachta indica, inhibits Plasmodium falciparum in culture. Southeast Asian J Trop Med Public Health 1985, 16:66-72.

22. Bray DH, Warhurst DC, Connolly JD, O'Neill MJ, Phillipson JD: Plants as sources of antimalarial drugs. Part 7. Activity of some species of Meliaceae plants and their constituent limonoids. Phytother Res 1990, 4:29-35.

23. Dhar R: Inhibition of the growth and development of asexual and sexual stages of drug sensitive and resistant strains of the human malaria parasite Plasmodium falciparum by neem (Azadiracta indica) fractions. J Ethnopharmacol 1998, 61:31-39.

24. Jones IW, Denholm AA, Ley SV, Lovell H, Wood A, Sinden RE: Sexual development of malaria parasites is inhibited in vitro by the neem extract azadirachtin, and its semi-synthetic analogues. FEMS Microbiol Lett 1994, 120:267-273.

25. Billker O, Shaw MK, Jones IW, Ley SV, Mordue AJ, Sinden RE: Azadirachtin disrupts formation of organised microtubule arrays during microgametogenesis of Plasmodium berghei. J Eukaryot Microbiol 2002, 49:489-497.

26. Boschitz C, Grunewald J: The effect of Neem Azal on Aedes aegypti (Diptera: Culicidae). Appl Parasitol 1994, 35:251-256

27. Mulla MS, Su TY: Activity and biological effects of neem products against arthropods of medical and veterinary importance. J Am Mosa Control Assoc 1999, 15:133-152.

28. Lucantoni L, Giusti F, Cristofaro M, Pasqualini L, Esposito F, Lupetti P, Habluetzel A: Effects of a neem extract on blood feeding, oviposition and oocyte ultrastructure in Anopheles stephensi Liston (Diptera: Culicidae). Tissue Cell 2006, 38:361-371.

29. Niemann L, Hilbig V: Neem seeds extracts as an example for health evaluation of naturally occurring substances to be applied in plant protection. Gesunde Pflanzen 2000, 52:135-141.

30. Udeinya IJ, Brown N, Shu EN, Udeinya Fl, Quakyi I: Fractions of an antimalarial neem leaf extract have activities superior to chloroquine, and are gametocytocidal. Ann Trop Med Parasitol 2006, 100:17-22.

31. Lamien CE, Meda A, Mans J, Romito M, Nacoulma OG, Viljoen GJ: Inhibition of fowlpox virus by an aqueous acetone extract from galls of Guiera senegalensis J. F. Gmel (Combretaceae). J Ethnopharmacol 2005, 96:249-253.

doi:10.1186/1756-3305-7-185

Cite this article as: Yerbanga et al:: Transmission blocking activity of Azadirachta indica and Guiera senegalensis extracts on the sporogonic development of Plasmodium falciparum field isolates in Anopheles coluzzii mosquitoes. Parasites \& Vectors 2014 7:185

\section{Submit your next manuscript to BioMed Central and take full advantage of:}

- Convenient online submission

- Thorough peer review

- No space constraints or color figure charges

- Immediate publication on acceptance

- Inclusion in PubMed, CAS, Scopus and Google Scholar

- Research which is freely available for redistribution

Submit your manuscript at www.biomedcentral.com/submit
C Biomed Central 